\title{
Tolerance and cross-tolerance to neurocognitive effects of THC and alcohol in heavy cannabis users
}

\author{
Johannes G. Ramaekers • Eef L. Theunissen • \\ Marjolein de Brouwer • Stefan W. Toennes • \\ Manfred R. Moeller • Gerhold Kauert
}

Received: 13 July 2010 / Accepted: 1 October 2010 / Published online: 30 October 2010

(C) The Author(s) 2010. This article is published with open access at Springerlink.com

\begin{abstract}
Introduction Previous research has shown that heavy cannabis users develop tolerance to the impairing effects of $\Delta 9$-tetrahydrocannabinol (THC) on neurocognitive functions. Animal studies suggest that chronic cannabis consumption may also produce cross-tolerance for the impairing effects of alcohol, but supportive data in humans is scarce.

Purpose The present study was designed to assess tolerance and cross-tolerance to the neurocognitive effects of THC and alcohol in heavy cannabis users.

Methods Twenty-one heavy cannabis users participated in a double-blind, placebo-controlled, three-way study. Subjects underwent three alcohol-dosing conditions that were designed to achieve a steady blood alcohol concentration of about $0,0.5$, and $0.7 \mathrm{mg} / \mathrm{ml}$ during a 5 -h time window. In addition, subjects smoked a THC cigarette $(400 \mu \mathrm{g} / \mathrm{kg})$ at $3 \mathrm{~h}$ post-onset of alcohol dosing during every alcohol condition. Performance tests were conducted repeatedly between 0 and $7 \mathrm{~h}$ after onset of drinking and included measures of perceptual motor control (critical tracking task), dual task processing (divided-attention task), motor
\end{abstract}

J. G. Ramaekers $(\bowtie) \cdot$ E. L. Theunissen $\cdot$ M. de Brouwer

Department Neuropsychology and Psychopharmacology,

Faculty of Psychology and Neuroscience, Maastricht University,

Maastricht, The Netherlands

e-mail: j.ramaekers@maastrichtuniversity.nl

S. W. Toennes $\cdot$ G. Kauert

Department of Forensic Toxicology, Institute of Legal Medicine,

Goethe University of Frankfurt,

Frankfurt, Germany

M. R. Moeller

Unikliniken des Saarlandes,

Homburg, Germany inhibition (stop-signal task), and cognition (Tower of London).

Results Alcohol significantly impaired critical tracking, divided attention, and stop-signal performance. THC generally did not affect task performance. However, combined effects of THC and alcohol on divided attention were bigger than those by alcohol alone.

Conclusion In conclusion, the present study generally confirms that heavy cannabis users develop tolerance to the impairing effects of THC on neurocognitive task performance. Yet, heavy cannabis users did not develop cross-tolerance to the impairing effects of alcohol, and the presence of the latter even selectively potentiated THC effects on measures of divided attention.

Keywords THC · Alcohol · Tolerance · Impulsivity · Cognition $\cdot$ Performance

\section{Introduction}

Cannabis use is largely concentrated among young people, aged 15-34 years. Population data suggest that, on average, $31 \%$ of young Europeans have ever used cannabis, while $12.5 \%$ have used the drug in the last year (EMCCDA 2009). In the USA, lifetime prevalence of cannabis use among young adults and last year prevalence are $49 \%$ and $21 \%$, respectively (DHHS/SAMHSA 2007). Prospective studies have demonstrated that despite spontaneous cessation of cannabis use in the majority of cannabis users, a substantial proportion of users develop stable use patterns characterized by continuous use of cannabis (Chen and Kandel 1995; Perkonigg et al. 2008; Perkonigg et al. 1999). It has been estimated that over $1 \%$ of all European adults, about 4 million, are using cannabis daily or almost daily. 
Most of these are aged 15-34 years, representing about $2.5 \%$ of Europeans in this age group (EMCCDA 2009).

Previous research has demonstrated that daily cannabis users are less sensitive to the impairing effects of $\Delta 9$ tetrahydrocannabinol (THC) intoxication on cognitive and psychomotor functions (D'Souza et al. 2008; Hart et al. 2001; Jones et al. 1981; Ramaekers et al. 2009) that have often been demonstrated in occasional cannabis smokers (Curran et al. 2002; Hart et al. 2002; Heishman et al. 1989; Lamers and Ramaekers 2001; Ramaekers et al. 2004; Ramaekers et al. 2006a), even when THC concentrations and levels of subjective high are similar (Ramaekers et al. 2009). This loss of sensitivity or tolerance to the behavioral effects of THC after prolonged use is believed to result from a change in pharmacodynamic response as evinced by CB1 receptor downregulation in large parts of the brain (Gonzalez et al. 2005). Alternatively, it has also been suggested that heavy cannabis users recruit alternative neural networks as a compensatory mechanism during task performance. Eldreth et al. (2004) and Kanayama et al. (2004) showed that compared with controls, cannabis users utilized additional brain regions to perform cognitive tasks, i.e., they compensated by working harder and recruiting compensatory networks.

Animal research has also suggested that pharmacological tolerance to the effects of THC may lead to cross-tolerance for actions of other drugs. Repeated cannabinoid administration decreased responsiveness of dopamine neurons in the mesoaccumbens in adolescent rats to an acute challenge with cannabinoid agonists but also to challenges with morphine, cocaine, and amphetamine (Pistis et al. 2004). The cannabinoid system has also been indicated in the development of tolerance to the effects of ethanol. Chronic ethanol exposure has been shown to produce downregulation of $\mathrm{CB} 1$ receptors and altered $\mathrm{CB} 1$ receptor gene expression (Hungund and Basavarajappa 2000; Ortiz et al. 2004). Rats made tolerant to the depressant effects of THC were also tolerant to the behavioral depressant effects of ethanol (Newman et al. 1972). Rats made tolerant to either ethanol or THC exhibited cross-tolerance to effects of the opposite compounds in learning and performance tasks (Siemens and Doyle 1979; Sprague and Craigmill 1976). These data strongly suggest the possibility of crosstolerance between ethanol and THC.

Ethanol and THC share many similarities in their actions. Pharmacological and behavioral effects of ethanol, such as hypothermia, euphoria, analgesia, sedation, and cognitive and motor dysfunction have also been demonstrated for THC (Ameri 1999; Iversen 2003). Combined use of ethanol and THC in occasional cannabis users has repeatedly been shown to increase the magnitude of cognitive and motor impairments in an additive manner (Lamers and Ramaekers 2001; Liguori et al. 2002;
Ramaekers et al. 2004). It is unclear however if combined use of ethanol and cannabis would also lead to similar impairments in these performance domains in heavy cannabis users. Based on the animal literature, it might be expected that heavy users of cannabis may develop tolerance to the impairing effects of THC, ethanol, and their combination. However, there is only little research in humans to support this claim. A few studies have reported (Casswell and Marks 1973; Marks and MacAvoy 1989; Wright and Terry 2002) that regular cannabis users were less impaired in peripheral signal detection and tracking accuracy than controls while intoxicated by THC and/or ethanol. These finding suggest either the development of tolerance and cross-tolerance in regular cannabis users, or their ability to compensate for intoxication effects.

The present study was designed to assess the effects of THC and alcohol, alone, and in combination, on neurocognitive performance of heavy cannabis users in order to establish the presence of tolerance or cross-tolerance to the impairing effects of THC and ethanol. Neurocognitive tasks were selected from previous studies demonstrating their sensitivity to measure THC-induced impairments in occasional cannabis users (Ramaekers et al. 2009; Ramaekers et al. 2006b).

\section{Methods}

\section{Subjects}

Twenty-one heavy cannabis users ( 15 males, 6 females) entered the present study. A summary of their demographics and history of drug use is given in Table 1. Subjects were recruited through advertisements in coffee shops. Initial screening comprised of a questionnaire on medical history. Subjects were examined by the medical supervisor who checked vital signs and took blood and urine samples. Standard blood chemistry, hematology, and drug screen tests were conducted on these samples. General inclusion criteria were: free from psychotropic medication; good physical health as determined by medical examination and laboratory analysis; absence of any major medical, endocrine, and neurological condition; normal weight, body mass index (weight per square length) between 18 and $28 \mathrm{~kg} / \mathrm{m}^{2}$; and written informed consent. Specific inclusion criteria were frequent use of cannabis (smoking on more than 4 days/week) during the previous year and presence of THC in serum on the day of screening. Exclusion criteria were: history of drug abuse (excluding marijuana) as assessed by drug urine screens and questionnaires; no experience with alcohol; non cigarette smokers; pregnancy or lactation or failure to use reliable contraceptives; color blindness, excessive drinking ( $>25$ standard alcoholic 
Table 1 Subject characteristics (mean, SD) and history of drug use for heavy cannabis users that completed the study $(N=19)$

Demographic variables
Age (years)

Age range (years)

Frequency of cannabis use/number of times per year

Joints per occasion (number)

History of cannabis use (years)

Frequency of alcohol use/number of times per year

Drinks per occasion

History of alcohol use (years)

Occasional use of other drugs (number of subjects)

\section{MDMA}

Amphetamine

Cocaine

LSD

Mushrooms

Salvia

Combined use of THC and alcohol (number of subjects)

Number of subjects attesting to driving under the influence of cannabis (DUIC)

Frequency of DUIC/year

Number of subjects attesting to driving under the influence of cannabis and alcohol (DUICA)

Frequency of DUICA/year

$23.2(8.4)$

$19-38$

373.7 (101.6)

$5.0(3.9)$

$9.0(5.5)$

$76.7(50.6)$

$8.4(5.7)$

$9.8(3.1)$

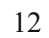

6

10

1

8

1

18

15

$139.5(172.9)$

6

$11.5(9.2)$ consumptions a week); hypertension (diastolic >100; systolic $>170$ ) or history of psychiatric disorders.

The study was conducted according to the code of ethics on human experimentation established by the declaration of Helsinki (1964) and amended in Seoul (2008). All subjects were fully informed of study procedures, adverse reactions to drug treatments, legal rights and responsibilities, expected benefits of a general scientific nature, and their right for voluntary termination without penalty or censure. A permit for obtaining, storing, and administering marijuana was obtained from the Dutch drug enforcement administration.

Design, doses, and administration

The study was conducted according to a double-blind, placebo-controlled, three-way design. Subjects underwent three alcohol-dosing conditions that were designed to achieve steady state blood alcohol concentration (BACs) of about $0,0.5$, and $0.7 \mathrm{mg} / \mathrm{ml}$ during a 5 -h time window. The order of alcohol-dosing conditions was counterbalanced across subjects. In addition, subjects smoked a THC cigarette $(400 \mu \mathrm{g} / \mathrm{kg})$ at $3 \mathrm{~h}$ post-onset of alcohol dosing, in each alcohol condition. Alcohol dosing started at 10:30 hours in the morning with placebo alcohol, 0.5 or
$0.7 \mathrm{~g} / \mathrm{kg}$ alcohol. Additional alcohol booster doses of about $0.1 \mathrm{~g} / \mathrm{kg}$ or alcohol placebo were given on an as needed basis at approximately every half hour up until $4.5 \mathrm{~h}$ after onset of alcohol dosing in order to keep BAC at the desired level. On average, subjects received 5.4 additional booster doses containing alcohol. Alcohol was administered as "pure" ethanol (96\%) mixed with orange juice to a volume of $300 \mathrm{ml}$ for the initial dose. Total volumes of booster doses mixed with orange juice were approximately $80 \mathrm{ml}$. THC smoking started at $3 \mathrm{~h}$ post-onset of alcohol dosing and lasted for about $15 \mathrm{~min}$. The cigarettes were prepared beforehand for each individual from stock provided by the Dutch Bureau for Medicinal Cannabis. Marijuana cigarettes were prepared from batches containing $11 \%$ THC, a standard potency for marijuana sold at Dutch pharmacies for medical use. The total amount of cannabis was weight calibrated for each individual subject and mixed with tobacco to achieve a standard cigarette size and weight. Subjects were instructed to smoke the cigarette according to a standardized procedure (Ramaekers et al. 2006a) in order to minimize the subject's possibility of dose titration and to increase optimal absorption of THC: i.e., inhale for $4 \mathrm{~s}$, hold breath for $10 \mathrm{~s}$, and exhale/break for $15 \mathrm{~s}$. This sequence was repeated until the cigarettes were smoked as completely as possible. Mean (SD) number of puffs smoked from the cigarette in the three alcohol/THC conditions were 17 (4.4), 17 (5.2), and 17 (2.9) respectively. A minimum wash-out of 4 days transpired between experimental treatments.

\section{Procedures}

Subjects were asked to refrain from drugs other than cannabis. Subjects were not allowed to use alcohol on the day prior to an experimental session and were requested to arrive at experimental sessions well rested. Subjects were allowed to continue their usual cannabis-smoking routine during the study period. Drug and alcohol screens were performed prior to experimental sessions upon arrival of the subject. Urine drug screens assessed for the presence of morphine, cocaine, marijuana, methampethamine, and amphetamine. Alcohol/THC treatments were only administered if subjects tested positive for THC, but negative for other drugs and alcohol. Subjects always tested positive for THC on test days. Subjects received a standardized lunch prior to THC smoking. Performance tests were conducted at fixed intervals during $7 \mathrm{~h}$ post-onset of alcohol dosing. The critical tracking task was conducted at $1 \mathrm{~h}, 2 \mathrm{~h}, 3 \mathrm{~h} 20,4 \mathrm{~h}$ $20 \mathrm{~min}, 5 \mathrm{~h} 20 \mathrm{~min}$ and $6 \mathrm{~h} 20 \mathrm{~min}$ post-onset of alcohol administration; a divided-attention task was conducted at $1 \mathrm{~h} 10 \mathrm{~min}, 3 \mathrm{~h} 30 \mathrm{~min}, 4 \mathrm{~h} 30 \mathrm{~min}$ and $5 \mathrm{~h} 30 \mathrm{~min}$ postonset alcohol dosing; the stop-signal task was conducted at $1 \mathrm{~h} 30 \mathrm{~min}, 4 \mathrm{~h}$ and $6 \mathrm{~h}$ post-onset alcohol dosing; and a 
Tower of London task was conducted at $1 \mathrm{~h} 40 \mathrm{~min}, 4 \mathrm{~h}$ $10 \mathrm{~min}$ and $6 \mathrm{~h} 10 \mathrm{~min}$ post-onset of alcohol dosing. Subjects received a training session prior to onset of the experimental sessions in order to familiarize them with the tests and procedures and minimize practice effects.

\section{Neurocognitive assessments}

The critical tracking test (CTT) measures the subject's ability to control a displayed error signal in a first-order compensatory tracking task. Error is displayed as a horizontal deviation of a cursor from the midpoint on a horizontal, linear scale. Compensatory joystick movements null the error by returning the cursor to the midpoint. The subject's compensatory response increases in frequency with an increasing phase lag. Control is lost at the point where the compensatory response lags the cursor's last movement by $180^{\circ}$. The response frequency at this point is defined as the critical frequency or lambda-c. The test includes five trials of which the lowest and the highest score are removed. The average of the remaining scores is taken as the final score (Jex et al. 1966)

The divided-attention task (DAT) measures the subject's ability to divide attention between two tasks performed simultaneously. The primary task consists of the tracking task as described above but at a constant level of difficulty set at $50 \%$ of the subject's maximum capacity. Tracking error is measured as the difference in millimeters between the position of the cursor and the midpoint of the scale. In the secondary task, the subject monitors a central display upon which single digits are presented at 1-s intervals. The occurrence of the digit " 2 " is a signal for the subject to remove the foot from a pedal as rapidly as possible. Inter stimulus interval varies between 1 and $2 \mathrm{~s}$. Mean absolute tracking error (millimeters) and number of control losses are the main parameters of the primary task. Number of correct signal detections and reaction time to signals are the main performance measures in the secondary task (Moskowitz 1973).

The stop-signal task (SST) measures motor impulsivity, which is defined as the inability to inhibit an activated or pre-cued response leading to errors of commission. The current test is adapted from an earlier version of Fillmore et al. (Fillmore et al. 2002) and has been validated for showing stimulant and sedative drug effects (Ramaekers and Kuypers 2006). The task requires subjects to make quick key responses to visual go signals, i.e., the letters ABCD presented one at a time in the middle of the screen, and to inhibit their response if a subsequent visual stop signal, i.e., "**, appears in one of the four corners of the screen. The stop signal is presented at predefined delays of $50,150,250$, and $350 \mathrm{msec}$. The main parameters are stop reaction time and commission errors during "no go" trials.
Stop reaction time represents the estimated mean time required to inhibit a response. Stop reaction time is calculated by subtracting the stop-signal delay from the reaction time on go-trials associated with $n$th percentile of the reaction time distribution. The $n$th percentile corresponds to the percentage of commission errors (Logan et al. 1984)

The Tower of London (TOL) is a decision-making task that measures executive function and planning (Shallice 1982). The original version of the Tower of London consists of three colored balls, which must be arranged on three sticks to match the target configuration on a picture while only one ball can be moved at a time. The present version consists of computer-generated images of beginand end-arrangements of the balls. The subject decides as quickly as possible, whether the end-arrangement can be accomplished in 2, 3, 4, or 5 steps from the begin arrangement by pushing the corresponding coded button. Number of correct decisions and mean reaction time are the main outcome measures.

\section{Subjective high and drunkenness}

Subjects rated their subjective high and drunkenness on visual analogue scales $(100 \mathrm{~mm})$ as a percentage of the maximum "high" or "drunkenness" ever experienced. Subjective high and drunkenness were rated on nine consecutive time points throughout $8 \mathrm{~h}$ after onset alcohol dosing. In addition, subject rated which of the two drugs (ethanol or THC) produced the most dominant feeling at each of these time points.

\section{Pharmacokinetic assessments}

Blood samples $(6 \mathrm{ml})$ were taken at baseline, 15, 30, 45 , and $60 \mathrm{~min}$ during the first hour after onset of $\mathrm{THC}$ smoking (THC cigarette was smoked within $15 \mathrm{~min}$ ) and subsequently at every $30 \mathrm{~min}$ between 1 and $4 \mathrm{~h}$ after smoking. Blood samples were centrifuged and serum was frozen at $-20^{\circ} \mathrm{C}$ until analyses for pharmacokinetic assessments. THC concentrations and its main metabolites (THC-COOH, OH-THC) were determined using a validated and accredited routine method for the analysis of cannabinoids in forensic blood samples (Toennes et al. 2008). The procedure essentially consists of an automated solid phase extraction and gas chromatography with mass spectrometric detection with a limit of quantification of $0.6 \mathrm{ng} / \mathrm{ml}$ which has also been successfully used for the analysis of THC in oral fluid (Kauert et al. 2006; Toennes et al. 2010). Subjects' BAC was monitored using a Lion SD4 breath alcohol analyser at baseline and approximately every $30 \mathrm{~min}$ through $8 \mathrm{~h}$ after onset of alcohol dosing. 
Statistics

All neurocognitive measures were analyzed with SPSS 13.0 using a GLM univariate analysis of variance with Alcohol (three levels) and THC over time (three to six levels, depending on the number of test repetitions) as fixed factors and Subjects as random factor. The univariate model tested for main effects of alcohol, THC over time, and alcohol $\times$ THC over time. The factor alcohol compares performance between three alcohol doses across all test replications and gives an indication of the overall effect of alcohol on performance. The factor THC over time compares performance before and after THC smoking across all alcohol conditions and gives an indication of the overall THC effect on performance. The interaction alcohol $\times \mathrm{THC}$ indicates whether the effect of THC on performance changes as a function of alcohol dose. Subjective measures of high and drunkenness were analyzed according to the same statistical design but tested only for the main effects of THC over time (nine levels) and alcohol (three levels), respectively.

\section{Results}

\section{Missing values}

Two subjects dropped out of the study after the first treatment condition for reasons unrelated to the study. Incomplete data from the drop-out subject did not enter statistical analysis. One subject was unable to reliably perform the divided-attention task. His data were not included in the statistical analysis of this task.

\section{BAC and THC concentrations}

Mean (SE) BAC concentrations and THC concentrations during treatments are shown in Fig. 1 and Table 2, respectively. On average, BAC concentration achieved the desired peak levels of 0.5 and $0.7 \mathrm{mg} / \mathrm{ml}$ in the low and high alcohol dose conditions. BAC levels dipped around $2.5 \mathrm{~h}$ after onset of dosing during lunch when subjects did not receive a booster dose, but returned to peak levels after administration of subsequent booster doses. BACs during the alcohol placebo condition were always zero. Concentrations of THC and its main metabolites were comparable in every treatment condition. Seven subjects indicated that they had smoked a cannabis cigarette on test days prior to the test session. All other subjects experienced their last cannabis cigarette during the preceding day. The impact of routine cannabis smoking of subjects was negligible since average baseline THC levels were low (i.e., $<10 \mathrm{ng} / \mathrm{mL}$ ) as compared to THC levels after smoking the experimental THC cigarette.
Subjective high and drunkenness

Subjective high was significantly elevated by the factor THC $\left(F_{8,462}=64.7 ; p=0.000\right)$. Subjective drunkenness was significantly elevated by alcohol $\left(F_{8,462}=86.6 ; p=0.000\right)$. Subjects indicated that the feeling of drunkenness was dominant prior to THC smoking and that the feeling of high was dominant after smoking. Mean (SE) rating of subjective high, drunkenness, and dominance of drug are shown in Fig. 2.

\section{Neurocognitive measures}

Lambda- $c$ in the critical tracking task significantly decreased after alcohol $\left(\mathrm{F}_{2,303}=5.42 ; p=0.005\right)$ but was not affected by THC or alcohol $\times$ THC. Mean (SE) lambda-c in every treatment condition is shown in Fig. 3.

Alcohol also significantly increased tracking error, control losses, and reaction time $\left(\mathrm{F}_{2,185}=6.68,9.51\right.$, and 16.91, respectively; $p<0.002$ ) and decreased the number of correct signal detections $\left(\mathrm{F}_{2,185}=7.6 ; p=0.001\right)$ in the divided-attention task. In addition, number of control losses, correct signal detections, and reaction time were also significantly affected by $\mathrm{THC}\left(\mathrm{F}_{3,185}=5.97,6.89\right.$, and 9.46, respectively; $p<0.001$ ). Control losses were also affected by the interaction of alcohol $\times$ THC $\left(\mathrm{F}_{6,185}=2.31\right.$; $p=0.036$ ). Mean (SE) performance in the divided-attention task in every treatment condition is shown in Fig. 4.

Stop reaction time $\left(\mathrm{F}_{2,141}=4.03 ; p=.020\right)$ and commission errors $\left(\mathrm{F}_{2,141}=7.51 ; p=0.001\right)$ in the stop-signal task significantly increased after alcohol, but were unaffected by THC or alcohol $\times$ THC. Performance in the Tower of London task was not affected by any factor. Mean (SE) performance in the stop-signal task in very treatment condition is shown in Fig. 5.

\section{Discussion}

The present study was designed to assess tolerance and cross-tolerance to the neurocognitive effects of THC and alcohol in heavy cannabis users. Results demonstrated that alcohol detrimentally affected performance of heavy cannabis users. THC generally did not affect performance, confirming earlier reports on tolerance to performance impairing effects of THC. Performance in the dividedattention task however was affected by both THC and alcohol, and their combination.

Alcohol was given in a low- and high-dose condition with the general aim to achieve steady BAC concentrations around 0.5 and $0.7 \mathrm{mg} / \mathrm{ml}$, respectively, during performance testing in a 5-h time window. After $5 \mathrm{~h}$, BACs were allowed to decline over time. Steady BACs were achieved by administering booster alcohol doses almost every $30 \mathrm{~min}$ 
Fig. 1 Mean (SE) BAC as a function of time after onset of alcohol drinking and onset of THC cigarette smoking in the low- and high-dose alcohol condition. Arrows indicate time points at which booster alcohol doses could be administered on an as needed basis to achieve steady BAC levels between 1 and $5 \mathrm{~h}$ after onset of drinking

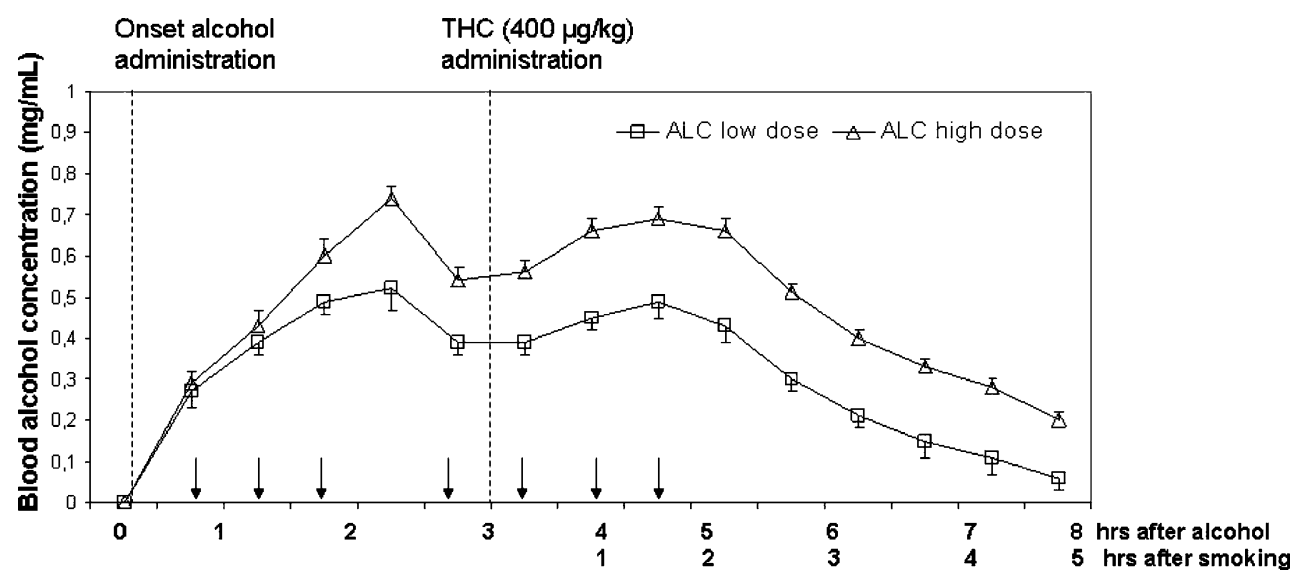

on an as needed basis. In general, repeated alcohol dosing produced the desired $\mathrm{BAC}$ concentrations during performance testing in the two alcohol conditions. Performance tests were basically scheduled in three separate time windows: i.e., between $1-2.5,3.5-5.5$, and 5.5-7 h after alcohol onset. Mean BAC concentrations in the low and high alcohol-dose condition fluctuated around 0.5 and $0.7 \mathrm{mg} / \mathrm{ml}$, respectively, during performance testing in the first and second time window. Performance testing during the third time window took place during the declining phase of BAC in both alcohol conditions. Subjective feelings of drunkenness during alcohol treatments were also comparable during the first and second time window, and gradually decreased with declining BACs in the third time window. BAC and subjective drunkenness data indicate that the levels of alcohol intoxication were comparable during performance testing in the first and second time window (i.e., prior and post-smoking THC),

Table 2 Mean (SD) serum concentrations of THC, THC-COOH, and $\mathrm{OH}-\mathrm{THC}$ as a function of time after onset of smoking

\begin{tabular}{|c|c|c|c|c|c|c|c|c|c|c|c|c|}
\hline & \multicolumn{12}{|c|}{ Time after smoking (h) } \\
\hline & & Baseline & 0.25 & 0.5 & 0.75 & 1 & 1.5 & 2 & 2.5 & 3 & 3.5 & 4 \\
\hline \multicolumn{13}{|c|}{ Alcohol placebo } \\
\hline \multirow[t]{2}{*}{$\mathrm{THC}$} & Mean & 8.6 & 112.1 & 49.3 & 32.0 & 24.7 & 20.7 & 13.4 & 10.1 & 8.8 & 8.6 & 7.9 \\
\hline & SD & 10.3 & 47.5 & 21.9 & 12.2 & 8.9 & 6.7 & 6.4 & 4.4 & 4.1 & 4.5 & 6.2 \\
\hline \multirow[t]{2}{*}{ THC-COOH } & Mean & 80.7 & 124.0 & 123.9 & 111.8 & 109.7 & 106.5 & 102.0 & 97.7 & 87.0 & 82.7 & 80.6 \\
\hline & $\mathrm{SD}$ & 75.1 & 102.4 & 104.6 & 91.3 & 91.8 & 90.4 & 84.9 & 86.7 & 74.3 & 64.7 & 67.0 \\
\hline \multirow[t]{2}{*}{ OH-THC } & Mean & 4.9 & 16.3 & 14.4 & 12.0 & 10.8 & 9.4 & 7.5 & 6.1 & 5.3 & 5.0 & 4.8 \\
\hline & $\mathrm{SD}$ & 7.1 & 10.0 & 10.0 & 7.8 & 6.8 & 5.5 & 4.6 & 4.4 & 3.8 & 3.9 & 3.8 \\
\hline \multicolumn{13}{|c|}{ Low alcohol dose } \\
\hline \multirow[t]{2}{*}{ THC } & Mean & 9.0 & 98.3 & 47.7 & 31.2 & 23.1 & 19.5 & 14.4 & 10.7 & 7.8 & 8.6 & 8.0 \\
\hline & $\mathrm{SD}$ & 11.8 & 50.0 & 28.0 & 19.1 & 13.2 & 12.2 & 9.2 & 7.4 & 5.1 & 6.3 & 6.0 \\
\hline \multirow[t]{2}{*}{ THC-COOH } & Mean & 58.6 & 82.9 & 83.7 & 79.0 & 75.7 & 76.3 & 70.4 & 65.6 & 52.3 & 57.3 & 54.0 \\
\hline & $\mathrm{SD}$ & 53.4 & 61.0 & 58.8 & 56.1 & 59.5 & 63.5 & 55.2 & 54.5 & 48.7 & 49.7 & 50.2 \\
\hline \multirow[t]{2}{*}{ OH-THC } & Mean & 4.5 & 18.4 & 15.2 & 12.6 & 10.6 & 9.8 & 7.7 & 6.1 & 4.7 & 5.0 & 4.3 \\
\hline & SD & 7.0 & 13.6 & 9.3 & 7.3 & 6.5 & 6.1 & 5.2 & 4.3 & 3.9 & 4.4 & 3.6 \\
\hline \multicolumn{13}{|c|}{ High alcohol dose } \\
\hline \multirow[t]{2}{*}{ THC } & Mean & 9.6 & 93.0 & 45.2 & 27.1 & 18.5 & 16.0 & 11.6 & 10.0 & 7.9 & 8.4 & 8.1 \\
\hline & $\mathrm{SD}$ & 15.7 & 40.5 & 26.7 & 14.2 & 8.6 & 7.8 & 6.3 & 5.9 & 5.1 & 5.4 & 6.0 \\
\hline \multirow[t]{2}{*}{ THC-COOH } & Mean & 70.3 & 87.9 & 96.7 & 94.2 & 85.8 & 73.1 & 71.0 & 68.8 & 62.9 & 60.1 & 62.3 \\
\hline & $\mathrm{SD}$ & 61.7 & 62.9 & 75.3 & 76.6 & 72.5 & 63.2 & 62.6 & 62.2 & 56.4 & 49.6 & 50.2 \\
\hline \multirow[t]{2}{*}{ OH-THC } & Mean & 5.8 & 17.8 & 16.1 & 12.8 & 10.8 & 8.8 & 7.3 & 6.5 & 5.2 & 5.2 & 4.8 \\
\hline & $\mathrm{SD}$ & 9.5 & 10.0 & 9.4 & 7.7 & 7.3 & 5.8 & 5.2 & 4.8 & 4.1 & 4.0 & 3.9 \\
\hline
\end{tabular}


Fig. 2 Mean (SE) subjective high (lower panel) and drunkenness (middle panel) as a function of time after alcohol and THC administration. The upper panel displays subjective dominance of alcohol or THC over time
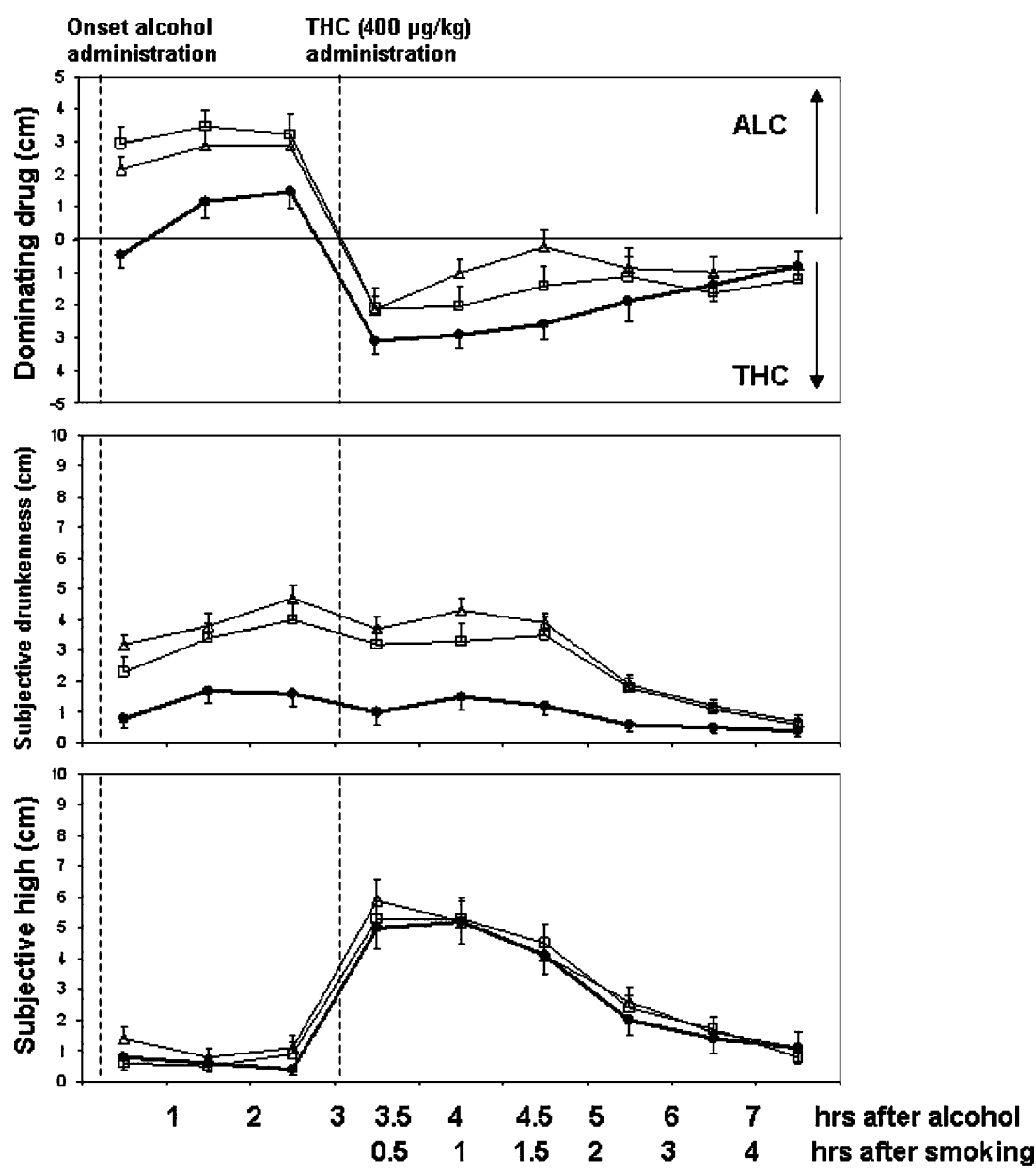

ALC placebo $\square$ ALC low dose $\triangle$ ALC high dose and declined at similar rates during performance assessments in the third time window.

The effects of alcohol in objective performance measures were consistent and straightforward. Alcohol significantly affected all performance measures in the critical tracking task, the divided-attention task, and the stop-signal task. In the critical tracking task, alcohol significantly decreased tracking performance. In the divided-attention task, alcohol increased reaction time, number of control losses, and decreased number correct signal detections and tracking. Alcohol increased stop reaction time and commission errors in the stop-signal task. The neurocognitive effects of alcohol in heavy cannabis users are comparable to those that have been reported in earlier studies in infrequent drug
Fig. 3 Mean (SE) lambda-c in the CTT as a function of time after alcohol and THC administration in every treatment condition

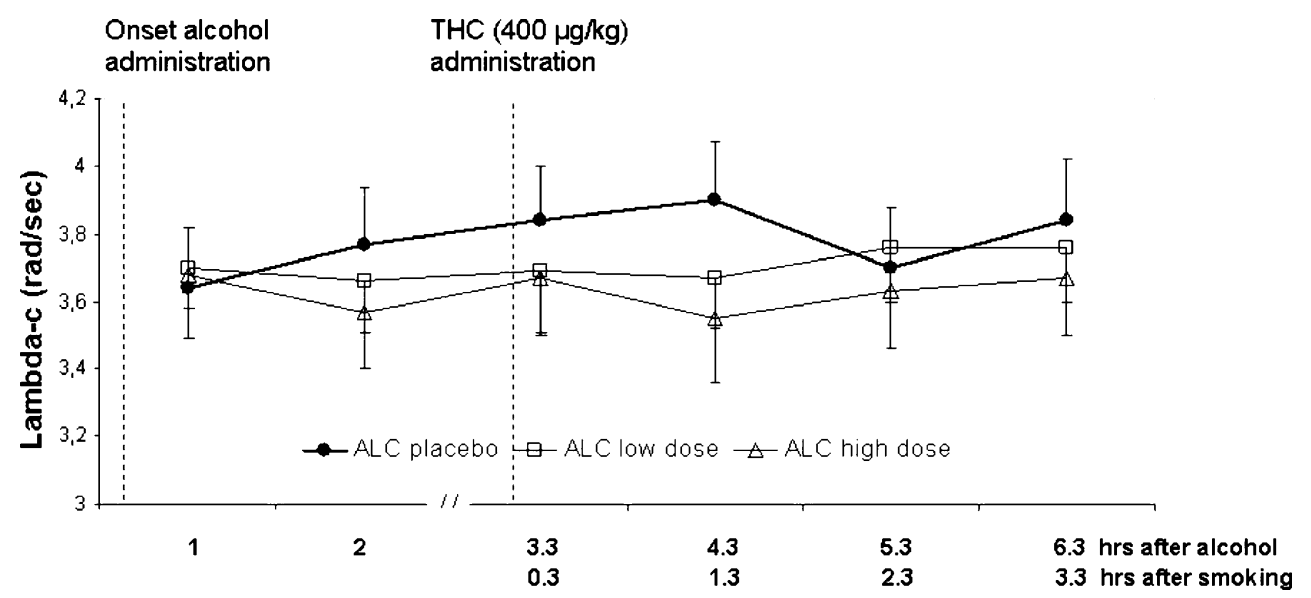



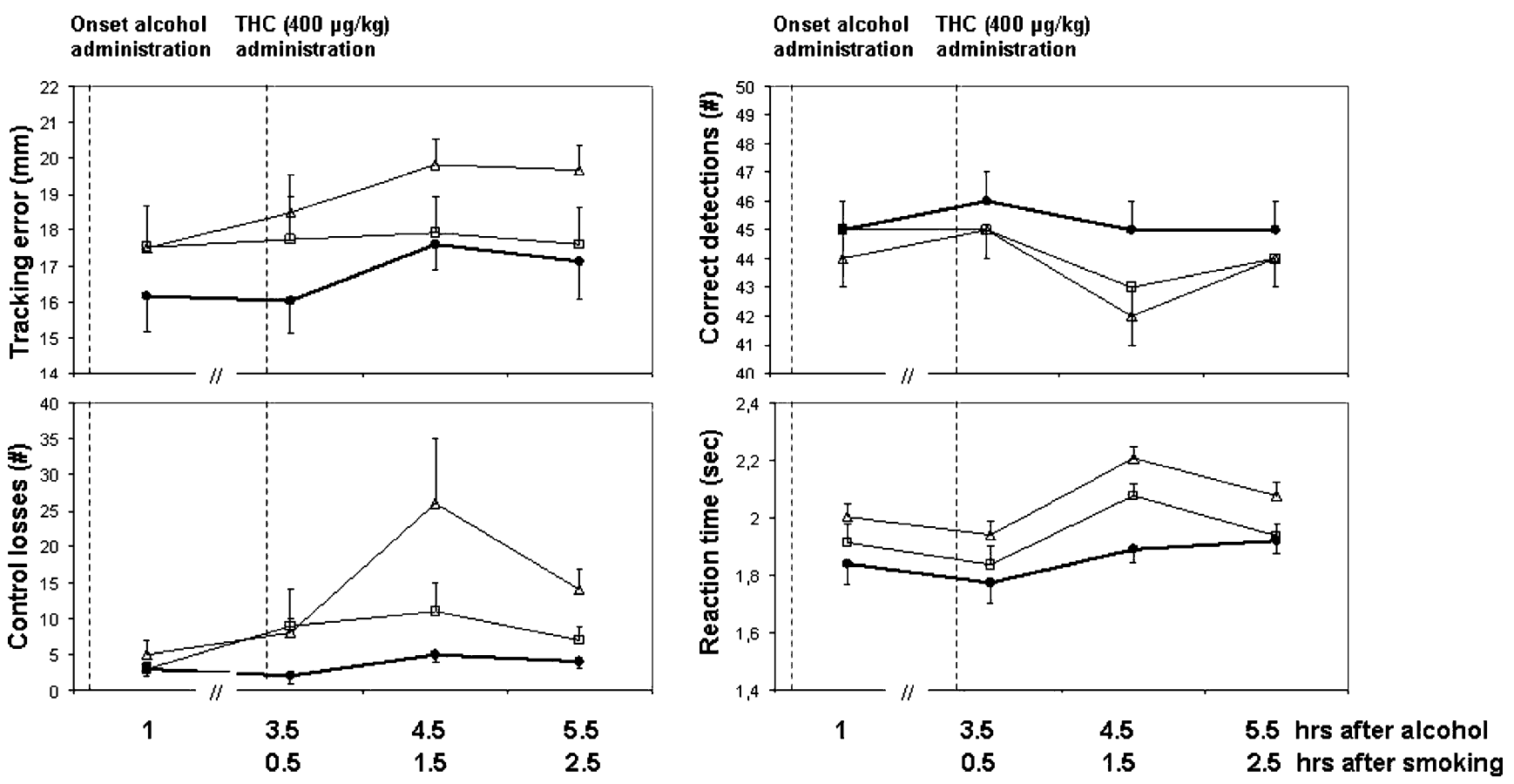

ALC placebo $\square$ ALC low dose $\triangle$ ALC high dose

Fig. 4 Mean (SE) tracking error, control losses, correct detections, and reaction time during the DAT as a function of time after alcohol and THC administration in every treatment conditions
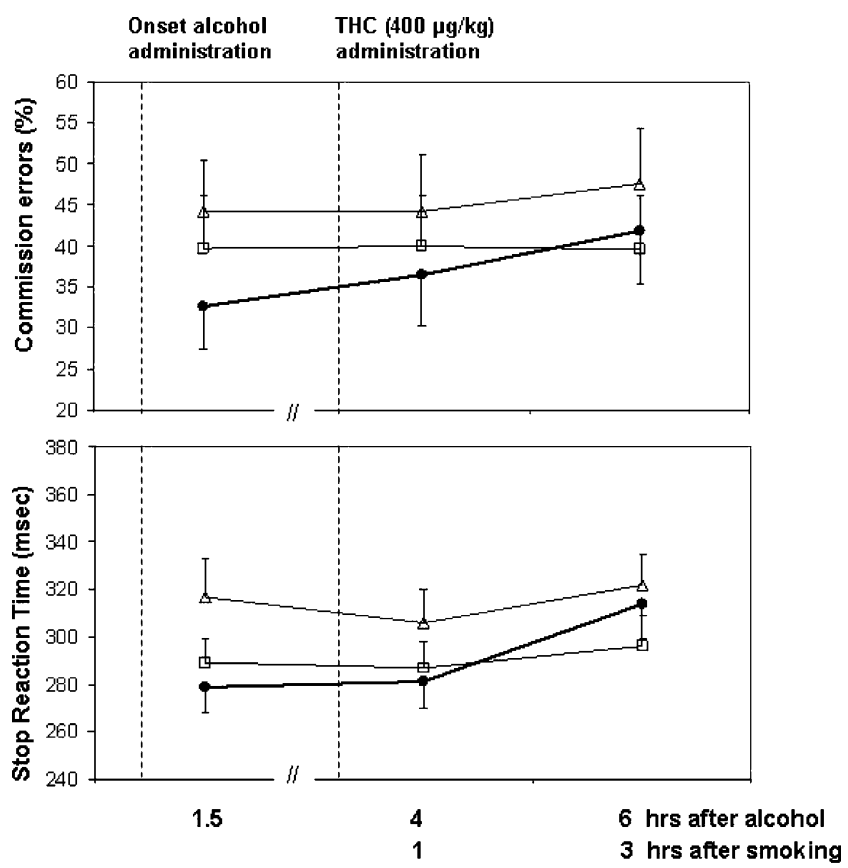

$\rightarrow$ ALC placebo $\square$ ALC low dose $\triangle$ ALC high dose

Fig. 5 Mean (SE) stop reaction time and commission errors in the SST as a function of time after alcohol and THC administration in every treatment condition (including cannabis) users (Heishman et al. 1988; Kuypers et al. 2006; Liguori et al. 2002; Ramaekers and Kuypers 2006) and healthy volunteers (de Wit et al. 2000; Vermeeren et al. 2002). The present data strongly indicate that heavy cannabis use does not produce cross-tolerance to the impairing potential of alcohol.

Subjects smoked a cannabis cigarette every treatment condition at $3 \mathrm{~h}$ after onset of alcohol or alcohol placebo administrations. Subjective high was elevated to similar degrees after smoking cannabis in each treatment condition. THC concentrations were also comparable between treatments with peak THC concentrations ranging from 93 to $112 \mathrm{ng} / \mathrm{ml}$. Together, these data suggest that THC administrations were very comparable in every treatment condition.

THC did not affect performance of heavy cannabis users in the critical tracking task, the stop-signal task, and the Tower of London. These tasks have previously been shown to be very sensitive to the impairing potential of THC when administered to infrequent cannabis (Ramaekers et al. 2006a). The lack of THC effects on any of these tasks basically confirms previous notions that heavy cannabis users can develop tolerance to behaviorally impairing effects of THC (D'Souza et al. 2008; Hart et al. 2001; Jones et al. 1981; Ramaekers et al. 2009). However it was interesting to note that tolerance was not apparent in all performance tasks. During dividedattention task performance, THC increased the number of control losses and reaction time and decreased the number of 
correct signal detections. Number of times that subjects lost control over the primary task (tracking) during this dual task performance appeared particularly sensitive to the impairing effect of THC. During alcohol placebo, mean number of control losses were always low, independent of $\mathrm{THC}$ administration. During treatments with low and high ethanol doses, mean number of control losses increased by a factor two and five, respectively, after smoking a THC cigarette. Univariate analysis indeed revealed a significant alcohol $\times$ THC interaction for this particular parameter, supporting the notion that the combination of alcohol and THC detrimentally affected the number of control losses in a synergistic manner.

THC effects on reaction time and signal detection in the divided-attention task may have also been related to concomitant alcohol use. The latter measures did not reveal an alcohol $\times$ THC interaction, but an additive effect of alcohol and THC cannot be excluded. Previous studies demonstrated that the divided-attention task is very sensitive to the effects of THC and alcohol alone when given to occasional cannabis users or healthy volunteers (Moskowitz 1984; Ramaekers et al. 2009; Schulte et al. 2001). Other studies have demonstrated that low doses of THC and alcohol that do not affect psychomotor function when given alone may still impair performance when given in combination (Lamers and Ramaekers 2001). In other words, small THC impairments that would go unnoticed in isolation still might exceed the (statistical) threshold of detection when added to the impairment produced by concurrent alcohol. Likewise, it is conceivable that negligible THC effects on divided attention as previously demonstrated in heavy cannabis users (Ramaekers et al. 2009) may become more apparent when added to those of a social dose of alcohol. This might particularly be true for attention tasks that are known for their very high sensitivity to drug and alcohol effects (Moskowitz 1984).

Data from the present study confirmed that chronic cannabis users develop tolerance to the behaviorally impairing effects of THC. However, previous notions (Marks and MacAvoy 1989; Wright and Terry 2002) that chronic cannabis use would also develop cross-tolerance for the impairing effects of alcohol were not confirmed. It should be noted however that previous studies never demonstrated complete tolerance to the behaviorally impairing effects of alcohol in heavy cannabis users. Generally, they showed that heavy cannabis users were less impaired after an alcohol challenge than non-drug users or infrequent cannabis users. Moreover, such demonstrations of partial tolerance were always very selective for single performance parameters (e.g., tracking accuracy), whereas other task parameters (e.g., reaction time) did not reveal cross-tolerance. Previous demonstrations of crosstolerance were obtained after administration of single doses of alcohol. Performance testing occurred during the descending phase of the blood alcohol curve. Consequently, BACs were generally lower than those obtained in the present study after repeated alcohol dosing. For example, Wright and Terry (2002) tested tracking performance of heavy cannabis users within $30 \mathrm{~min}$ after drinking while mean BACs declined from 0.28 to $0.22 \mathrm{mg} / \mathrm{ml}$. Mean BAC levels in the present study however were two to three times as high and experimentally controlled to achieve relatively steady state levels during $5 \mathrm{~h}$ of repeated performance testing. Repeated alcohol challenges and high BAC levels thus may have provoked more pronounced alcohol impairments than can be observed after single administration of a low alcohol dose. Consequently, cross-tolerance or behavioral adaptation may have been lacking or insufficient to compensate for prolonged alcohol impairments as observed in the present study.

The general lack of cross-tolerance for the impairing effects of alcohol as well as the potential of ethanol to potentiate the effects of THC in the divided-attention task may have important implications for heavy cannabis users who drive under the influence of both drugs. Heavy cannabis users usually operate their vehicle on day to day basis because they believe they developed resistance against the impairing effect of THC (Ramaekers et al. 2009). In the present study, most participants (79\%) admitted to driving under the influence of cannabis and a substantial proportion (32\%) also admitted to driving under the influence of cannabis and alcohol in combination. The present data however demonstrated that mean BAC concentrations up to $0.7 \mathrm{mg} / \mathrm{ml}$ produce significant performance impairment and that the presence of alcohol may potentiate detrimental effects of THC during dual task performances that are common during car driving. Additive and synergistic effects of alcohol and $\mathrm{THC}$ on driving performance have previously been shown in occasional cannabis users (O'Kane et al. 2002; Ramaekers et al. 2004; Sewell et al. 2009). The present study demonstrates that additive and synergistic effects of THC and alcohol on performance can pertain to heavy cannabis users as well.

In conclusion, the present study generally confirms that heavy cannabis users develop tolerance to the impairing effects of THC on neurocognitive task performance. Yet, heavy cannabis users did not develop cross-tolerance to the impairing effects of alcohol, and the presence of the latter even selectively potentiated THC effects on measures of divided attention.

Acknowledgements We would like to express our gratitude to Bea Krause, Nathalie Valle-Guzman, Sanne van Rooij, and Lisa Schoenfeld for their assistance in data collection.

Disclosure This study was supported by a grant from the German Society against Alcohol, Drugs, and Driving ("Bund gegen Alkohol und Drogen im Straßenverkehr"). 
Open Access This article is distributed under the terms of the Creative Commons Attribution Noncommercial License which permits any noncommercial use, distribution, and reproduction in any medium, provided the original author(s) and source are credited.

\section{References}

Ameri A (1999) The effects of cannabinoids on the brain. Prog Neurobiol 58:315-348

Casswell S, Marks D (1973) Cannabis induced impairment of performance of a divided attention task. Nature 241:60-61

Chen K, Kandel DB (1995) The natural history of drug use from adolescence to the mid-thirties in a general population sample. Am J Public Health 85:41-47

Curran HV, Brignell C, Fletcher S, Middleton P, Henry J (2002) Cognitive and subjective dose-response effects of acute oral delta 9-tetrahydrocannabinol (THC) in infrequent cannabis users. Psychopharmacology (Berl) 164:61-70

de Wit H, Crean J, Richards JB (2000) Effects of d-amphetamine and ethanol on a measure of behavioral inhibition in humans. Behav Neurosci 114:830-837

DHHS/SAMHSA (2007) Results from the 2007 National Survey of Drug Use and Health: National findings, pp 1-294

D'Souza DC, Ranganathan M, Braley G, Gueorguieva R, Zimolo Z, Cooper T, Perry E, Krystal J (2008) Blunted psychotomimetic and amnestic effects of Delta-9-tetrahydrocannabinol in frequent users of cannabis. Neuropsychopharmacology 33:2505-2516

Eldreth DA, Matochik JA, Cadet JL, Bolla KI (2004) Abnormal brain activity in prefrontal brain regions in abstinent marijuana users. Neuroimage 23:914-920

EMCCDA (2009) Annual report 2009: the state of the drugs problem in Europe

Fillmore MT, Rush CR, Hays L (2002) Acute effects of oral cocaine on inhibitory control of behavior in humans. Drug Alcohol Depend 67:157-167

Gonzalez S, Cebeira M, Fernandez-Ruiz J (2005) Cannabinoid tolerance and dependence: a review of studies in laboratory animals. Pharmacol Biochem Behav 81:300-318

Hart CL, van Gorp W, Haney M, Foltin RW, Fischman MW (2001) Effects of acute smoked marijuana on complex cognitive performance. Neuropsychopharmacology 25:757-765

Hart CL, Ward AS, Haney M, Comer SD, Foltin RW, Fischman MW (2002) Comparison of smoked marijuana and oral Delta(9)tetrahydrocannabinol in humans. Psychopharmacology (Berl) 164:407-415

Heishman SJ, Stitzer ML, Bigelow GE (1988) Alcohol and marijuana: comparative dose effect profiles in humans. Pharmacol Biochem Behav 31:649-655

Heishman SJ, Stitzer ML, Yingling JE (1989) Effects of tetrahydrocannabinol content on marijuana smoking behavior, subjective reports, and performance. Pharmacol Biochem Behav 34:173-179

Hungund BL, Basavarajappa BS (2000) Are anandamide and cannabinoid receptors involved in ethanol tolerance? A review of the evidence. Alcohol Alcohol 35:126-133

Iversen L (2003) Cannabis and the brain. Brain 126:1252-1270

Jex HR, McDonnell JD, Phatak AV (1966) A "critical" tracking task for man-machine research related to the operator's effective delay time. I. Theory and experiments with a first- order divergent controlled element. NASA CR-616. NASA Contract Rep NASA CR: 1-105

Jones RT, Benowitz NL, Herning RI (1981) Clinical relevance of cannabis tolerance and dependence. J Clin Pharmacol 21:143 S-152 S

Kanayama G, Rogowska J, Pope HG, Gruber SA, Yurgelun-Todd DA (2004) Spatial working memory in heavy cannabis users: a functional magnetic resonance imaging study. Psychopharmacology (Berl) 176:239-247

Kauert GF, Iwersen-Bergmann S, Toennes SW (2006) Assay of Delta9-tetrahydrocannabinol (THC) in oral fluid-evaluation of the OraSure oral specimen collection device. J Anal Toxicol 30:274-277

Kuypers KP, Samyn N, Ramaekers JG (2006) MDMA and alcohol effects, combined and alone, on objective and subjective measures of actual driving performance and psychomotor function. Psychopharmacology (Berl) 187:467-475

Lamers CT, Ramaekers JG (2001) Visual search and urban driving under the influence of marijuana and alcohol. Hum Psychopharmacol 16:393-401

Liguori A, Gatto CP, Jarrett DB (2002) Separate and combined effects of marijuana and alcohol on mood, equilibrium and simulated driving. Psychopharmacology (Berl) 163:399-405

Logan GD, Cowan WB, Davis KA (1984) On the ability to inhibit simple and choice reaction time responses: a model and a method. J Exp Psychol Hum Percept Perform 10:276-291

Marks DF, MacAvoy MG (1989) Divided attention performance in cannabis users and non-users following alcohol and cannabis separately and in combination. Psychopharmacology (Berl) 99:397-401

Moskowitz H (1973) Laboratory studies of the effects of alcohol on some variables related to driving. J Saf Res 5:185-192

Moskowitz H (1984) Attention tasks as skills performance measures of drug effects. Br J Clin Pharmacol 18(Suppl 1):51 S-61 S

Newman LM, Lutz MP, Gould MH, Domino EF (1972) 9 Tetrahydrocannabinol and ethyl alcohol: evidence for crosstolerance in the rat. Science 175:1022-1023

O'Kane CJ, Tutt DC, Bauer LA (2002) Cannabis and driving: a new perspective. Emerg Med (Fremantle) 14:296-303

Ortiz S, Oliva JM, Perez-Rial S, Palomo T, Manzanares J (2004) Chronic ethanol consumption regulates cannabinoid CB1 receptor gene expression in selected regions of rat brain. Alcohol Alcohol 39:88-92

Perkonigg A, Lieb R, Hofler M, Schuster P, Sonntag H, Wittchen HU (1999) Patterns of cannabis use, abuse and dependence over time: incidence, progression and stability in a sample of 1228 adolescents. Addiction 94:1663-1678

Perkonigg A, Goodwin RD, Fiedler A, Behrendt S, Beesdo K, Lieb R, Wittchen HU (2008) The natural course of cannabis use, abuse and dependence during the first decades of life. Addiction 103:439-449, discussion 450-1

Pistis M, Perra S, Pillolla G, Melis M, Muntoni AL, Gessa GL (2004) Adolescent exposure to cannabinoids induces long-lasting changes in the response to drugs of abuse of rat midbrain dopamine neurons. Biol Psychiatry 56:86-94

Ramaekers JG, Kuypers KP (2006) Acute effects of 3, 4methylenedioxymethamphetamine (MDMA) on behavioral measures of impulsivity: alone and in combination with alcohol. Neuropsychopharmacology 31:1048-1055

Ramaekers JG, Berghaus G, van Laar M, Drummer OH (2004) Dose related risk of motor vehicle crashes after cannabis use. Drug Alcohol Depend 73:109-119

Ramaekers JG, Kauert G, van Ruitenbeek P, Theunissen EL, Schneider E, Moeller MR (2006a) High-Potency Marijuana Impairs Executive Function and Inhibitory Motor Control. Neuropsychopharmacology 31:2296-2303. doi:10.1038/sj.npp.1301068

Ramaekers JG, Moeller MR, van Ruitenbeek P, Theunissen EL, Schneider E, Kauert G (2006b) Cognition and motor control as a function of Delta9-THC concentration in serum and oral fluid: limits of impairment. Drug Alcohol Depend 85:114 122

Ramaekers JG, Kauert G, Theunissen EL, Toennes SW, Moeller MR (2009) Neurocognitive performance during acute THC intoxica- 
tion in heavy and occasional cannabis users. J Psychopharmacol 23:266-277

Schulte T, Muller-Oehring EM, Strasburger H, Warzel H, Sabel BA (2001) Acute effects of alcohol on divided and covert attention in men. Psychopharmacology (Berl) 154:61-69

Sewell RA, Poling J, Sofuoglu M (2009) The effect of cannabis compared with alcohol on driving. Am J Addict 18:185-193

Shallice T (1982) Specific impairments of planning. Phil Trans of the Royal Society of London 298:199-209

Siemens AJ, Doyle OL (1979) Cross-tolerance between delta9tetrahydrocannabinol and ethanol: the role of drug disposition. Pharmacol Biochem Behav 10:49-55

Sprague GL, Craigmill AL (1976) Ethanol and delta-9tetrahydrocannabinol: mechanism for cross-tolerance in mice. Pharmacol Biochem Behav 5:409-415
Toennes SW, Ramaekers JG, Theunissen EL, Moeller MR, Kauert GF (2008) Comparison of cannabinoid pharmacokinetic properties in occasional and heavy users smoking a marijuana or placebo joint. J Anal Toxicol 32:470-477

Toennes SW, Ramaekers JG, Theunissen EL, Moeller MR, Kauert GF (2010) Pharmacokinetic properties of delta-9-tetrahydrocannabinol in oral fluid of occasional and chronic users. J Anal Toxicol 34 (4):216-21

Vermeeren A, Riedel WJ, van Boxtel MP, Darwish M, Paty I, Patat A (2002) Differential residual effects of zaleplon and zopiclone on actual driving: a comparison with a low dose of alcohol. Sleep 25:224-231

Wright A, Terry P (2002) Modulation of the effects of alcohol on driving-related psychomotor skills by chronic exposure to cannabis. Psychopharmacology (Berl) 160:213-219 